\title{
Transitions Between Periodic Orbits and Control of Chaos Assisted by an External Force in Hamiltonian Systems
}

\author{
R.M. Angelo, K. Furuya ${ }^{\dagger}$ and M.A.M. de Aguiar ${ }^{\ddagger}$ \\ Instituto de Física, 'Gleb Wataghin' \\ Universidade Estadual de Campinas, UNICAMP \\ Caixa Postal 6165 - CEP: 13081-970, Campinas, SP, Brazil
}

Received 22 April, 1998

\begin{abstract}
We investigate the possibility of inducing transitions between periodic orbits in twodimensional Hamiltonian systems by means of a time-localized external perturbation. We show that the amplitude of the perturbation can be approximately calculated in the limit of a delta-type force in terms of the initial and final periodic orbits. For a specific Hamiltonian, we show several numerical examples where the external perturbation, varied from delta-type to gaussian, allows transitions between specifically chosen members of families of periodic orbits. The same mechanism is then applied to move aperiodic chaotic orbits into periodic ones, presenting a new way to control chaotic behavior in Hamiltonian systems.
\end{abstract}

\section{Introduction}

Hamiltonian systems with two or more degrees of freedom are known to be generically non-integrable. In most cases the phase space shows a complex mixture of regular and chaotic regions, both permeated densely by periodic orbits. These orbits, as pointed out by Poincaré [1], play a crucial role in the theory of dynamical systems. Since each orbit is completely specified by its initial conditions, it is possible to map the phase space into regular and chaotic zones and launch the system in any desired phase space region. In practical situations however, like that of an electric circuit [2] or cardiac systems [3], the precise initial conditions are not available and, as the system is turned on, it jumps to one of a large variety of possible states, many of them corresponding to chaotic orbits. It is sometimes very important to force the system to a particular nonchaotic state, usually a periodic orbit. In this sense, controlling chaotic systems has become an important issue in a variety of scientific disciplines [4]. Several ad- vancements and new methods have been proposed [5] since the seminal paper by Ott, Grebogy and Yorke [6] (the $O G Y$ method). Most of these methods were envisaged to move an orbit embedded in a chaotic attractor to an unstable periodic orbit and are applicable only to dissipative systems. The basic idea to achieve this goal is to apply small and periodic perturbations to the dynamical system, chosen according to which periodic orbit one wants the system to move to.

Despite the enormous amount of work devoted to applications and developments on control of chaos in dissipative systems, very little has been done in this direction for conservative systems. One possible extension of the OGY method for conservative maps was proposed by Lai et al [7], which has been used to construct rough orbits that achieve a desired transport rapidly [8], also called time optimal control, or targeting. The aim of this paper is to investigate an alternative mechanism to induce transitions between trajectories in Hamiltonian systems. Our interest here is not exclusively on the control of chaos, but rather on the possibility of

\footnotetext{
*Electronic address: renato@ifi.unicamp.br

$\dagger$ Electronic address: furuya@ifi.unicamp.br

$\ddagger$ Electronic address: aguiar@ifi.unicamp.br
} 
induce transitions between two pre-specified trajectories, especially periodic orbits. We shall restrict our attention to non-integrable systems with two degrees of freedom. Although we focus here mainly on transitions between two periodic orbits, we show that transitions from non-periodic chaotic orbits to periodic orbits can be performed exactly in the same way, indicating a new direction for controlling chaos in Hamiltonian systems. Contrary to the OGY-type procedures, our method is not based on the application of small periodic perturbations to the original system, but on a single appropriate perturbation induced by a time localized external field, constituting what we call a single kick targeting.

This paper is organized as follows: in sec.II we introduce our main ideas considering initially the effect of a simple delta-like kick, given at an appropriate time and with calculated intensity, on inducing the desired transition between two periodic orbits. We then allow for a more realist external perturbation in the form of a gaussian pulse with controllable width. In section (III) we exemplify the method with a particular Hamiltonian system, the two degrees of freedom non-integrable Nelson Hamiltonian. We show several examples of transitions between periodic orbits, and in particular a transition from a non-periodic chaotic orbit to a periodic one. In section IV we make some concluding remarks.

\section{The Method}

Let $H\left(x, y, p_{x}, p_{y}\right)$ be the Hamiltonian of a system with two freedoms and $\mathcal{O}^{i}$ and $\mathcal{O}^{t}$ two periodic solutions. The indices $i$ and $t$ stand for initial and target respectively. One possible way to promote a particle from $\mathcal{O}^{i}$ to $\mathcal{O}^{t}$ is by kicking the orbit with appropriate forces. Suppose the particle is at the point $\left(x^{i}, y^{i}, p_{x}^{i}, p_{y}^{i}\right)$, belonging to $\mathcal{O}^{i}$, at time $t_{0}$ and we want it to go to $\left(x^{t}, y^{t}, p_{x}^{t}, p_{y}^{t}\right)$, belonging to $\mathcal{O}^{t}$. The addition of a perturbation of the form $\alpha_{x} p_{x} \delta\left(t-t_{0}\right)$ to the Hamiltonian would change Hamilton's equation for $\dot{x}$ into

$$
\dot{x}=\frac{\partial H}{\partial p_{x}}+\alpha_{x} \delta\left(t-t_{0}\right) .
$$

Choosing $\alpha_{x}=\left(x^{t}-x^{i}\right)$ has the effect of adjusting the $x$ coordinate by just the amount required to put it on the desired point of $\mathcal{O}^{t}$. Similar terms would give appropriate kicks in $y, p_{x}$ and $p_{y}$. Of course that would be a rather complicated, if not practically impossible, way to implement the transition, since it would require four independent kicks at the same time. In what follows we describe a method that reduces this complication to a single kick and we give numerical examples of its realization. This single delta-like impulse will be replaced later by a gaussian, so that the external force acting on the particle can be interpreted as, say, an electric field that is turned on and off smoothly.

The whole idea is very simple, and in order to achieve our goal, we make the initial assumption that the initial orbit $\mathcal{O}^{i}$ crosses (in coordinate space) the target orbit $\mathcal{O}^{t}$. We shall denote the coordinates and the momenta by two-vectors $X=(x, y)$ and $P=\left(p_{x}, p_{y}\right)$ respectively. Let this crossing point be at $X=\alpha$. Therefore, kicking the orbit at $X=\alpha$ eliminates the need for two of the four parameters initially necessary. We are left with the two momenta to work with. Let $P=\rho^{i}=\left(\rho_{x}^{i}, \rho_{y}^{i}\right)$ be the momenta of $\mathcal{O}^{i}$ at $X=\alpha$. The energy of the orbit is given by $E^{i}=\rho^{i^{2}} / 2+V(\alpha)$ and the velocity $\dot{X}$ makes an angle $\theta^{i}$ with the $x$-axis given by $\tan \theta^{i}=\dot{y} / \dot{x}=\rho_{y}^{i} / \rho_{x}^{i}$. For the target orbit, on the other hand, the velocity points in a different direction $\theta^{t}$. If $\Delta \theta=\left|\theta^{t}-\theta^{i}\right|<\pi / 2$, we can align the two velocities with a single kick in, say, the momentum $p_{y}$. Indeed, the addition of the term $A y \delta\left(t-t_{0}\right)$ to the Hamiltonian produces a change $\rho_{y}^{i} \rightarrow \rho_{y}^{i}-A$ that puts the velocity in the desired direction if $A$ is chosen to be $\left(\rho_{x}^{t} \rho_{y}^{i}-\rho_{x}^{i} \rho_{y}^{t}\right) / \rho_{x}^{t}$.

The total energy after the kick is $E^{f}=\gamma \rho^{t^{2}} / 2+$ $V(\alpha)$ where $\gamma=\left(\rho_{x}^{i} / \rho_{x}^{t}\right)^{2}$. Therefore, the change in the particle's total energy is $\Delta^{f i}=E^{f}-E^{i}=\gamma \rho^{t^{2}}-\rho^{i^{2}}$ and we also compute $\Delta^{f t}=E^{f}-E^{t}=(\gamma-1) \rho_{2}^{2}$, the difference between the actual energy after the kick and the energy of the target orbit. Clearly we could not launch the particle exactly onto a chosen new trajectory by adjusting a single parameter. Even requiring the two trajectories to cross each other in coordinate space, tuning the direction of the momentum will change its modulus and vice-versa. However, in most situations this change in energy is not crucial: one is not interested in jumping exactly to a particular orbit $\mathcal{O}^{t}$, but rather in jumping to some member of the $\mathcal{O}^{t}$ periodic orbit family, at an appropriate energy. (It is well known that the periodic orbits of a Hamiltonian system, without explicit time-dependence occur in one-dimensional families of varying energy [1], [9].) 
In the next section we shall see numerical examples where this simple procedure works well in inducing transitions. The basic reason for this success is that, in those cases, the member of the target family to which the original orbit is being moved to, crosses it at an angle that depends only weakly on the energy of the member. Therefore, even if the change $\Delta^{f t}$ is not small, the orbit is launched on a direction that still corresponds approximately to that of a periodic orbit. In some cases, however, the angle between the orbit velocities changes appreciably with the energy and the kick fails to move the orbit to the desired target. To contemplate these cases without changing the basic idea discussed above, we replace the delta-like kick by a smooth gaussian perturbation centered not necessarily at the crossing point, but slightly before or after that, as illustrated, for example, in Fig. (1.d). This modification improves considerably the performance of the method, as we shall show in section III . Therefore, we define the perturbative potential to be $\delta_{\epsilon}\left(t-t_{0}\right) y$ or $\delta_{\epsilon}\left(t-t_{0}\right) x$, depending on whether we want the force to act in the $y$ or $x$ direction, with

$$
\delta_{\epsilon}=A\left[\frac{1}{\epsilon \sqrt{\pi}} \exp \left[-\left(\frac{t-t_{o}}{\epsilon}\right)^{2}\right]\right]
$$

where $A$ measures the amplitude of the perturbation and $t_{0}$ and $\epsilon$ are the center and the width of the gaussian respectively.

\section{EXAMPLES}

The examples to be discussed in this section are all from the Nelson Hamiltonian [9]

$$
H_{\mathcal{N}}=\frac{1}{2}\left(p_{x}^{2}+p_{y}^{2}\right)+\left(y-\frac{1}{2} x^{2}\right)^{2}+\mu \frac{x^{2}}{2} .
$$

where $\mu$ is a parameter that we shall fix at $\mu=0.1$.

At low energies one can disregard the cubic and quartic terms of $H_{\mathcal{N}}$ and get a 2-D harmonic oscillator with frequencies $\sqrt{2}$ in the $y$-direction and $\sqrt{\mu}$ in the $x$-direction. As the energy is increased the periodic solutions corresponding to the $x$-modes (or horizontal modes) couple with the $y$ coordinate (the vertical direction) and bend upwards in the form of a wide 'u'. The harmonic oscillation in the vertical direction continues to be an exact solution due to the symmetry of the Hamiltonian. As the energy is further increased, both periodic orbit families, that we call $H$, for Horizontal, and $V$, for Vertical, undergo multiple bifurcations generating several other families. The families of periodic orbits of this system and their bifurcations are well studied and a detailed account can be found in refs. $[9,10]$.

At high energies the periodic orbits of the Nelson Hamiltonian have complicated shapes when plotted in the $x-y$ coordinate space. Nevertheless, several families can still be classified as vertical, in the sense that their oscillations are wider in the $y$-direction than in the $x$-direction. It is clear that an external force in the $y$-direction should be useful to jump from nearly horizontal periodic orbits to these essentially vertical ones. We shall see now that this is indeed the case. In order to indicate the stability character of the periodic orbits we add the letter $S$ (for stable) or U (for unstable) at the end of the family name.

\section{External force in the $y$-direction}

With the gaussian perturbation, the potential becomes:

$$
V(x, y)=\left(y-\frac{x^{2}}{2}\right)+\mu \frac{x^{2}}{2}+y A \delta_{\epsilon}\left(t-t_{0}\right)
$$

The equations of motion are :

$$
\begin{aligned}
& \dot{x}=\frac{\partial H}{\partial p_{x}}=p_{x} \\
& \dot{y}=\frac{\partial H}{\partial p_{y}}=p_{y} \\
& \dot{p_{x}}=-\frac{\partial H}{\partial x}=2 x\left(y-\frac{x^{2}}{2}\right)-\mu x \\
& \dot{p_{y}}=-\frac{\partial H}{\partial y}=2\left(y-\frac{x^{2}}{2}\right)-A \delta_{\epsilon}\left(t-t_{o}\right) .
\end{aligned}
$$

As discussed in section II, we are going to switch on the perturbation when the initial orbit approaches the target orbit in coordinate space. For periodic librations (self-retracing orbits) the crossing points are traversed twice and we choose one of the two in order to have the best agreement between the values of $p_{x}$ on the original and target orbits. The variable $p_{y}$ will be impacted via the adjustment of the perturbation potential parameters and it is the most affected of the variables if $\epsilon$ is small.

To estimate $A$ we first suppose that $\epsilon=0$. Integrating the equations above about $t=t_{0}$ produces a shift in the momentum $p_{y}$ of magnitude $\delta p_{y}=-A$. As discussed in section II we need to set $A$ to $\left(\rho_{x}^{t} \rho_{y}^{i}-\rho_{x}^{i} \rho_{y}^{t}\right) / \rho_{x}^{t}$. 
In the case of a Horizontal initial orbit we can approximate $\rho_{y}^{i}=0$ and get

$$
A=-\rho_{x}^{i} \rho_{y}^{t} / \rho_{x}^{t}=-\rho_{x}^{i} \tan \theta^{t}
$$

Fig. 1(a) shows an example where the delta kick works well. In this case a member of the Horizontal family HS at energy $E^{i}=0.0048$ is moved into a member of the family V3U (see Table I) with $E^{t}=0.0943$. We plotted separately in fig 1(a1) the original periodic orbit HS and the target orbit V3U, while in Fig. 1(a2) we show the transition itself $\mathrm{HS} \rightarrow \mathrm{V} 3 \mathrm{U}$. The circle in part (a1) shows the position where the perturbation is turned on.

TABLE $\mathbf{I}: \mathbf{H} \rightarrow \mathrm{V}$ and $\mathbf{H} \rightarrow \mathbf{H}$ transitions

\begin{tabular}{||c|c|c|c|c|c|c||}
\hline In. Family & $A$ & $\epsilon$ & Fi. Family & $\left|A_{\text {cale }}\right|$ & $\Delta^{f t}$ & Figure \\
\hline HS & -0.43 & 0.01 & V3U & -0.4 & 0.0089 & $1 .(\mathrm{a})$ \\
HS & -0.25 & 0.01 & V4U & -0.2 & 0.0047 & $1 .(\mathrm{b})$ \\
HS & 0.7 & 1.0 & V4S & - & 0.0028 & $1 .(\mathrm{c})$ \\
HS & -1.283 & 1.0 & V4U & - & 0.2335 & $1 .(\mathrm{d})$ \\
\hline \hline IS & 0.6 & 0.57 & V4S & 0.36 & 0.0065 & $2 .(\mathrm{a})$ \\
IS & 0.5283 & 0.18 & HS & 0.44 & 0.0095 & $2 .(\mathrm{b})$ \\
IS & 0.428 & 0.80 & IS & 0.55 & 0.0063 & $2 .(\mathrm{c})$ \\
\hline \hline
\end{tabular}

TABLE II : $\mathbf{V} \rightarrow \mathbf{H}$ and $\mathbf{V} \rightarrow \mathbf{V}$ transitions

\begin{tabular}{||c|c|c|c|c|c|c||}
\hline In. Family & $A$ & $\epsilon$ & Fi. Family & $\left|A_{\text {cale }}\right|$ & $\Delta^{f t}$ & Figure \\
\hline V4S & -0.57 & 0.1 & HU & 0.25 & 0.0077 & $3 .(\mathrm{a})$ \\
V4S & -0.706 & 0.4 & IU & 0.41 & 0.0101 & $3 .(\mathrm{b})$ \\
V3S & 0.4880 & 0.02 & IU & - & 0.0193 & $3 .(\mathrm{c})$ \\
V4S & -0.533 & 0.1 & V4S & - & 0.0178 & $3 .(\mathrm{d})$ \\
\hline
\end{tabular}

Using the values of $\rho_{x}^{i} \simeq \sqrt{2 E^{i}} \simeq 0.1$ and $\tan \theta^{t} \simeq$ -4 gives $A=-0.4$. The actual parameters used in this transition were $A=-0.43$ and $\epsilon=0.01$. The time step used in the numerical integration is typically $\delta t=0.01$ and the perturbation has been applied in such a way that the center of the gaussian sits on one of the steps of integration, no longer being effective at the next step, therefore simulating a delta function. In this case, the target orbit has period $\tau=13.05$ and is slightly unstable: the Lyapunov exponent is approximately 0.024 . Therefore, we cannot expect the final trajectory to stay in its neighborhood for times longer than about $3 \tau$.

Fig. 1(b) shows another example of transition induced by a delta-like perturbation, namely $\mathrm{HS} \rightarrow \mathrm{V} 4 \mathrm{U}$. In this case $A$ is estimated to be -0.2 and the actual value used was $A=-0.25$. As in part (a), this Fig. (and the others to come) have two parts, displayed side by side: part 1 contains the original periodic orbits (the one to be perturbed and the one to be reached) with a circle indicating the kick position. The transition itself is shown in part 2, so that the degree of success in each case can be seen clearly. In Table I we display the numerical values of the parameters used in each case.

Figs. (1c) and (1d) show examples of transitions where the gaussian perturbation is used. The original orbit is the same as in (1a) and (1b) and the target orbits are $\mathrm{V} 4 \mathrm{~S}$ an $\mathrm{V} 4 \mathrm{U}$ respectively. The time-width used in both cases is $\epsilon=1.0$. It is important to notice that these cases include a ' $\mathrm{S}$ ' (stable) and a ' $\mathrm{U}$ '(unstable) final periodic orbits. 

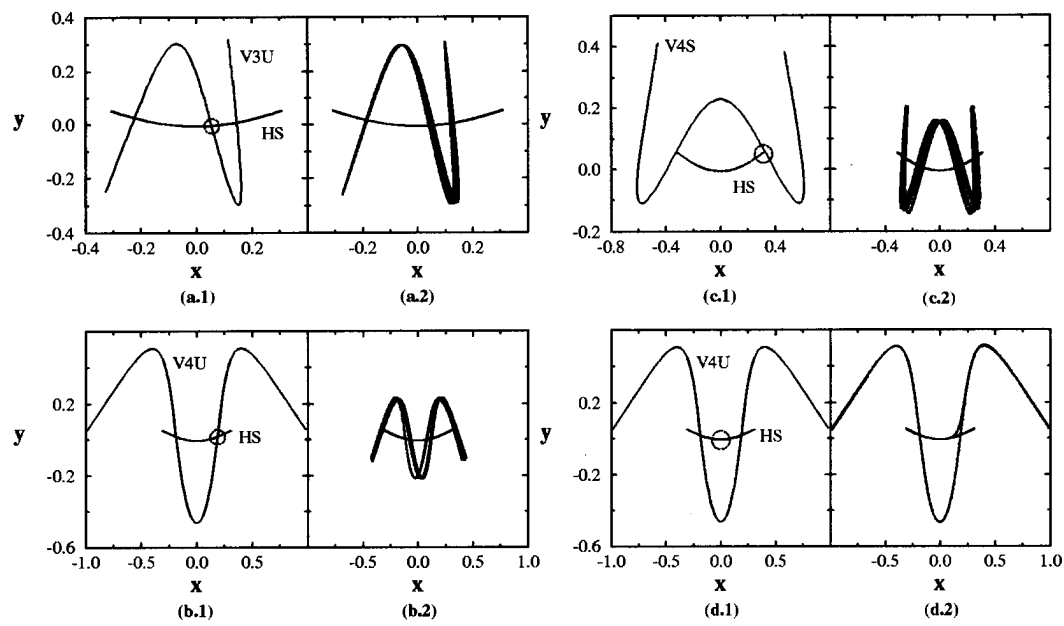

Figure 1. (a1)Initial p.o. of the HS family with $E^{i}=0.0048$ and target p.o. of the V3a family with $E=0.0975$. (a2) The transition from the initial p.o. of the HS family to an orbit of the V3U family with energy $E^{t}=0.0943$, with $A=-0.43$ and delta kick (total time elapsed: $t=100$ ); (b1) Initial p.o. of the family HS and the target p.o. of the family V4U with $E=0.2349$, (b2) $\mathrm{H} \rightarrow \mathrm{V} 4 \mathrm{U}$ with lower final energy (total time elapsed: $\mathrm{t}=100$ ); (c1)Initial p.o. of the family HS and the target p.o. of the V4S family with $E=0.1058$, (c2) HS $\rightarrow$ V4S (total time elapsed: $t=100) ;(d 1)$ Initial p.o. of the HS family and the target p.o. of the V4U family with $E=0.2349,(\mathrm{~d} 2) \mathrm{HS} \rightarrow \mathrm{V} 4 \mathrm{U}$ (total time elapsed: $\mathrm{t}=47$ ).
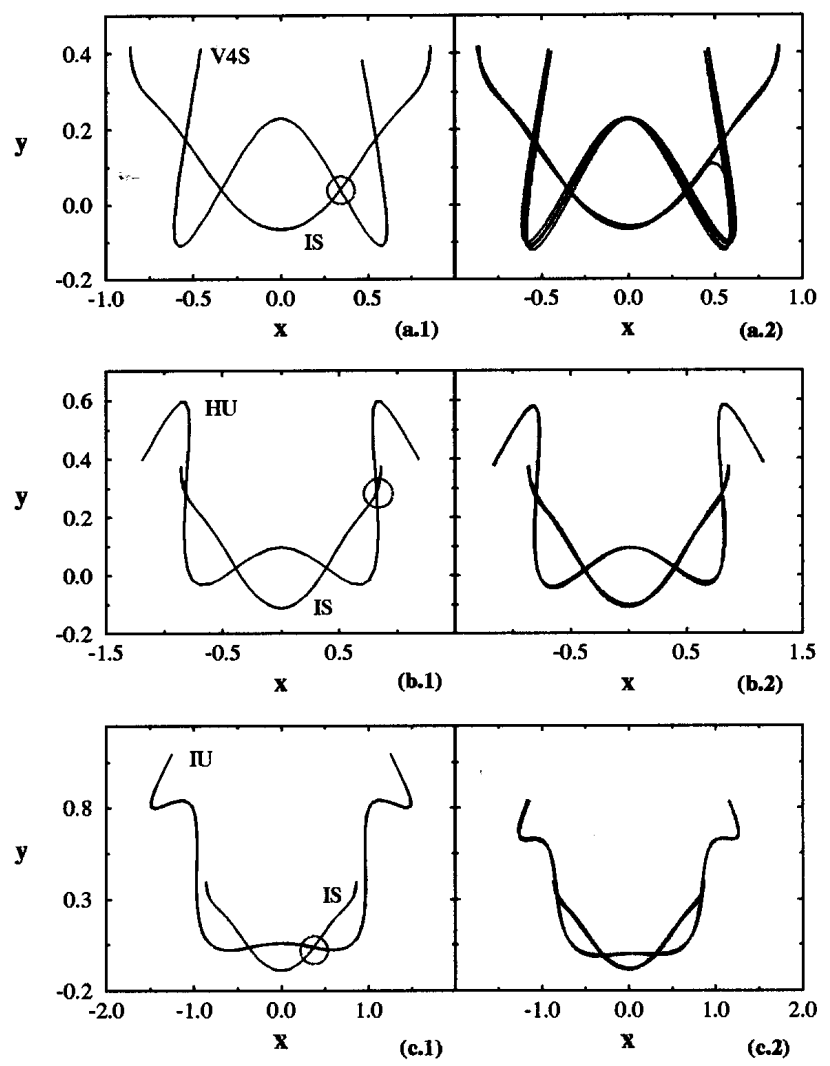

Figure 2. Transitions with the gaussian modulated force in the $y$-direction. The initial p.o. in all cases belongs to the I family with $E^{i}=0.0394$. (a1) Initial p.o. of the IS family and target p.o. of the V4S family with $E=0.1058$, (a2) IS $\rightarrow$ V4S (total time elapsed: $\mathrm{t}=100$ ); (b1) Initial p.o. of the IS family and target p.o. of the HU family with $E=0.1370,(\mathrm{~b} 2)$ IS $\rightarrow$ HU (total time elapsed: $\mathrm{t}=100$ ); (c1) Initial p.o. of the IS family and target p.o. of the same IU family with $E=0.1026$ , (c2) IS $\rightarrow$ IU (total time elapsed: $t=100$ ). See cases 6 to 8 of table I for the parameter values. 
Figs. 2(a) and 2(b) show transitions at higher energies from the IS family to V4S and HS respectively, and Fig. 2(c) shows a transition IS $\rightarrow$ IS between two members of the I family at $E=0.03943$ and $E^{t}=0.1026$.

So, using the above-described procedure and making a judicious choice of parameters for the perturbation term, we have been able to make the transition from horizontal to vertical periodic orbits on the Nelson Hamiltonian given in Eq.(1) above.

\section{External force in the $x$-direction}

For completeness we have also studied the reverse case, from a Vertical family to a Horizontal family, which is also possible if we apply the force accordingly. Since the same type of reasoning is involved, we just show in Table II a few transitions in order to illustrate this case.

Parts (a) and (b) of Fig. 3 show transitions from V4S to HU and IU respectively. Part (c) shows a transition V3S to IU and part (d) shows a transition between different members of the same family, namely V4S.
Finally, having guaranteed the successful application of this method for transitions between periodic orbits (stable and unstable), we have also considered the possibility of jumping from non-periodic chaotic trajectories to the vicinity of a specific stable periodic orbit. It turns out that it is indeed possible to produce such transitions and the level of difficulty is the same as in the case of periodic orbits. We illustrate this case taking the chaotic trajectory shown in Fig. 4a, and ending up in each one of the p.o. of the I family shown in the inset of Figs. 4b (IS) and 4c (IU). These cases $C \rightarrow$ p.o. are shown in Figs. 4b and 4c. In Fig. 4b the final torus is near the p.o. of the IS family with energy $E^{t}=0.0447$, which is lower than the initial energy. In order to satisfy the condition given by Eq.(5) for $E^{t}<E^{i}$, we have to choose the crossing point (or kicking region) such that:

$$
\left|p_{y o}\right| \geq \sqrt{2\left(E^{i}-E^{t}\right)}
$$

In Fig. 4c we show another case where the final p.o. is in the IU family with energy $E^{t}=0.1858$.
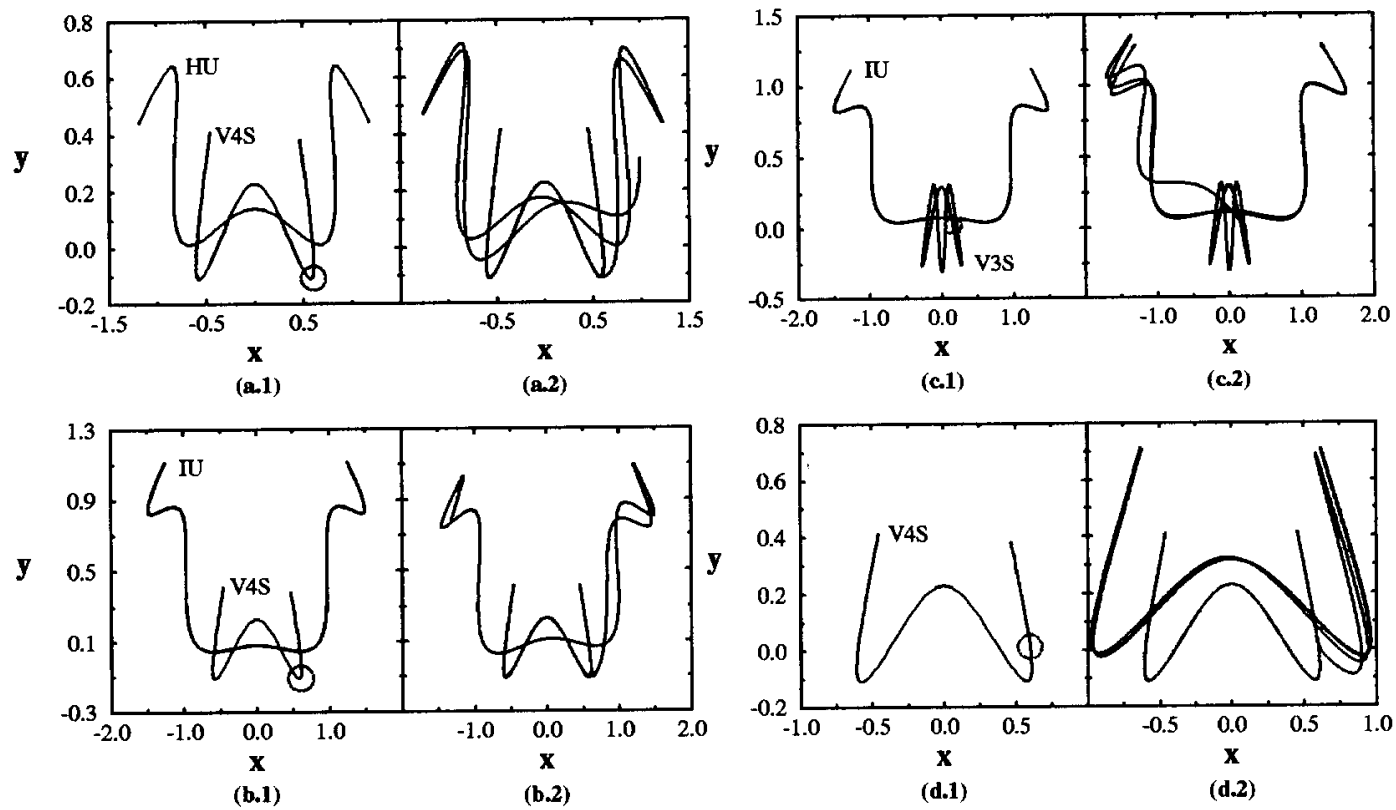

Figure 3. Transitions with the gaussian force in the $x$-direction. The initial periodic orbit V4S family has $E^{i}=0.1058$ and V3S has $E^{i}=0.0962$. (a1)target p.o. from HU family with $E=0.1370$, (a2) V4S $\rightarrow$ HU (total time elapsed: t=40); (b1)target p.o. of the IU family with $E=0.1895$, (b2) V4S $\rightarrow$ IU (total time elapsed: t=40); (c1) Initial p.o. from the V3S family with $E^{i}=0.0962$ and target p.o. from the IU family with $E=0.1370$, (c2) V3S $\rightarrow$ IU (total time elapsed: t=70); (d1) Initial p.o. from the V4S family with $E^{i}=0.1058$ and target p.o. from the same family with higher energy (d2) V4S $\rightarrow$ V4S (total time elapsed: $t=100$ ). See table II for the parameter values. 


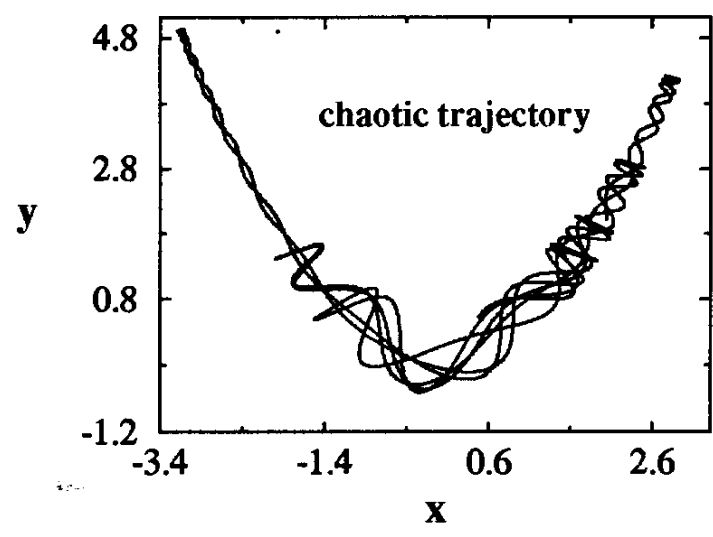

(a)
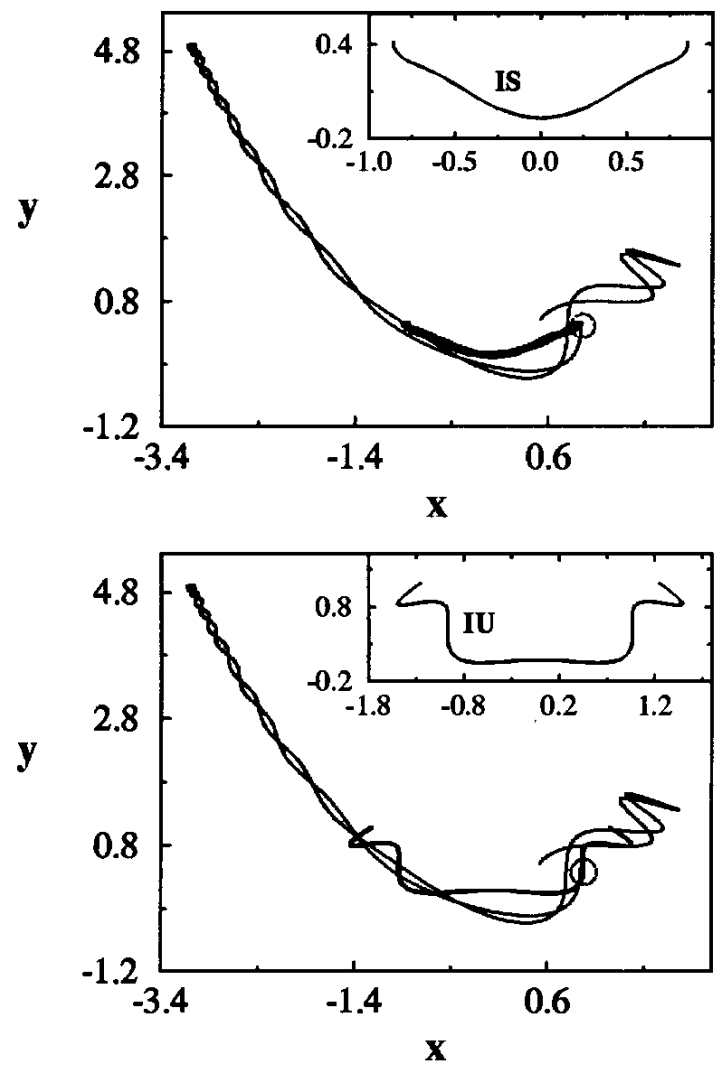

(c)

Figure 4. The transition from a non-periodic chaotic trajectory (C) with energy $E^{i}=0.5039$ to periodic orbits. (a) the chaotic trajectory evolved up to $t=100$; (b) transition from the chaotic trajectory to a torus close to the p.o. of the IS family with energy $E^{t}=0.0447, A=1.0000$ and $\epsilon=0.01$. (c) transition from the chaotic trajectory to the p.o. of the IU family with energy $E^{t}=0.1858, A=1.4918$ and $\epsilon=0.01$; In all cases the total evolution time $t=100$.

\section{Concluding Remarks}

The gaussian-modulated perturbations as in Eq.(1) have proved to be very effective to perform p.o-p.o. transitions, including aperiodic chaotic $(\mathrm{C})$ to periodic orbits. Several transitions have been obtained by means of such a smooth time-localized perturbation, namely:
$\mathrm{H} \rightarrow \mathrm{V}, \mathrm{H} \rightarrow \mathrm{H}, \mathrm{V} \rightarrow \mathrm{H}, \mathrm{V} \rightarrow \mathrm{V}$ and $\mathrm{C} \rightarrow \mathrm{H}$ orbits of the Nelson Hamiltonian (Eq.(2)) taken as a testing ground. All the transitions happened with some change in energy, as expected, due to the time-dependent kick. The time intervals where the transitions effectively occur have turned out to be very short compared to the periods of the periodic orbits of the Nelson system. In some cases, the time width $\epsilon$ of the perturbation was of the order of the step of numerical integration $(\delta t=0.01)$, and the perturbation acted essentially like a delta-function kick. To illustrate how the jump in energy occurs, we plot one case in Fig. 5 which refers to the transition of Fig. 2(a), with $\epsilon=0.57$.

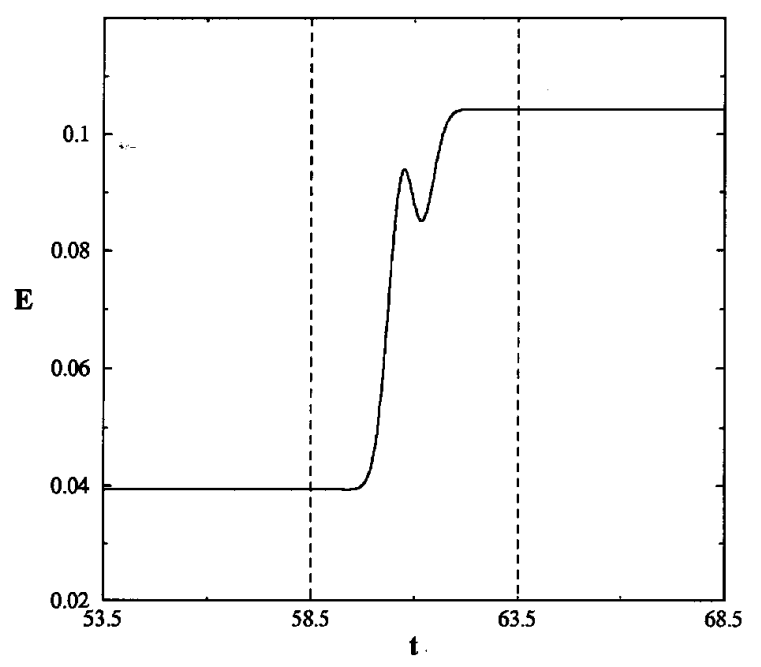

Figure 5. The time evolution of the energy during a shorttime kick with $\epsilon=0.57$, much larger than the integration step $\delta t=0.01$, corresponding to the case of Fig.2(a).

Although the method proposed here is general and may be applied to any two-degree-of-freedom nonintegrable Hamiltonian system, it is crucial that the initial trajectory and the final p.o. actually cross each other in the $x y$-plane. We point out the importance of having a detailed study of the main families of periodic orbits of the system in question: without such knowledge, it becomes impossible to induce the transitions. Finally, we note that this is still a preliminary study as far as control of chaos is concerned. Although we have demonstrated the feasibility of the single kick targetting, we have not derived the conditions under which a chaotic trajectory would be moved to the neighborhood of a desired periodic orbit given that only a limited knowledge of the system is available. This would 
be a very important result if the present method is to be applied in practical situations.

\section{Acknowledgments}

The authors acknowledge helpful discussions at the beginning of this work with N. Rahman. We also thank M. Baranger for a critical reading of the manuscript and for several suggestions. This work has been partly supported by FAPESP, CNPq and FINEP.

\section{References}

[1] H. Poincaré, Les methodes nouvelles de la mechanique céleste (Gauthier-Villars, Paris, 1892), vol. I, chap. III, Art. 36.

[2] T. Matsumoto and L.O. Chua, IEEE Trans. Circuits. Syst., CAS-32, 797 (1985); K. Murali and M. Lakshmanan, Int. J. Bifurcation and Chaos 2, 621 (1992).

[3] L. Glass, Phys. Today 49, 40 (1996); Hall K, Christini DJ, Tremblay M, et al., Phys. Rev. Lett. 78, 4518 (1997).
[4] F. Muller, Ecol. Model 100, 135 (1997); Earn DJD, Rohani P, Grenfell BT, P. Roy. Soc. Lond. B BIO 265, 7 (1998); Schroer CG, Ott E, Chaos 7, 512 (1997); Ding MZ et al., Chaos 7, 644 (1997); Petrov V, Showalter K, Chaos 7, 614 (1997).

[5] E.Ott, C.Greborgi and J.A. York, 'Controlling chaotic dynamical systems',in: D Campbell (Ed.) Chaos/XAOC, Soviet-American Perspective on Nonlinear Science, American Institute of Physics,1990, New York; A. Huebbler, Helvetica Phys. Acta 62, 343 (1989); K. Pyragas, Phys. Lett. A170, 421 (1992).

[6] E. Ott, C. Grebogi and J.A. York, Phys. Rev. Lett. 64, 1196 (1990).

[7] Y-C. Lai, M. Ding and C. Grebogi, Phys. Rev. E47, 86 (1993).

[8] E.M. Bollt, J.D. Meiss, Physica D 81, 280 (1995); idem Phys. Lett. A204, 373 (1995).

[9] M. Baranger and K.T.R. Davies, Ann. Phys. 177, 330 (1987).

[10] M. A. M. de Aguiar, C. P. Malta, M. Baranger and K. T. R. Davies, Ann. Phys. 180, 167 (1987). 